\title{
Case Report: Posterior Ischemic Optic Neuropathy
}

\author{
Hyukjin Kwon, OD \\ You \& Eye Optical Inc. \\ Sarah MacIver, OD, FAAO \\ School of Optometry \\ \& Vision Science \\ University of Waterloo
}

\begin{abstract}
\section{BACKGROUND}

Posterior ischemic optic neuropathy (PION) is a vascular disorder that occurs in the posterior part of the optic nerve and therefore usually does not present with optic disc edema.
\end{abstract}

\section{CASE REPORT}

We report here a case of PION with an uncharacteristic presentation. It highlights the importance of understanding the different pathophysiologies of PION and anterior ischemic optic neuropathy (AION) to help differentiate between the two clinically, and includes a literature review of PION.

\section{KEY WORDS:}

Posterior Ischemic Optic Neuropathy (PION), pial vascular plexus, posterior ciliary arteries (PCA), Watershed zone

\section{INTRODUCTION}

Ischemic optic neuropathies are often differentiated based on the location of the ischemia and the etiology. Anterior ischemic optic neuropathy (AION) involves the anterior $1 \mathrm{~mm}$ of the optic nerve head and often presents with edema at the optic disc, resulting in visible optic nerve swelling. AION can be further classified based on its etiology; non-arteritic (NA-AION) or arteritic (A-AION), which is almost always associated with giant cell arteritis. Posterior ischemic optic neuropathy (PION) is an ischemia that presents at any location behind the optic disc and, by definition, does not cause optic nerve swelling., PION is a diagnosis of exclusion and is more rare than AION. There are overlapping characteristics between AION and PION, including systemic risk factors and sudden painless vision loss. We can differentiate between AION and PION clinically if we thoroughly understand the pathophysiologies of the diseases. Currently, different treatment options are being investigated and systemic corticosteroids seem to show promising results for both conditions. ${ }^{3,4}$

We present here a case that highlights the importance of understanding the pathogenesis and clinical presentation of PION to help differentiate PION from AION. Understanding the different types of ischemic optic neuropathies is important for the primary eye care provider to provide prompt and appropriate treatment for patients. 


\section{CASE REPORT}

A 60-year-old South Asian woman presented for an ocular emergency exam. The patient's chief complaint was neck pain that preceded sudden vision loss OS. The patient reported that diffuse neck pain on the left side started approximately one week previously and vision loss began 3 days previously. At the exam, she reported worsening vision, mild pain in the eye (3 out of 10), frontal headache and photophobia. The patient denied any redness, discharge, jaw claudication, scalp tenderness, fever, malaise or history of recent ocular trauma or surgery. She denied flashes or floaters in her vision. Her medical history was positive for hypothyroidism and medication included Synthroid.

Presenting aided visual acuity was $6 / 6^{+2} \mathrm{OD}$ and $6 / 21^{-2}$ OS at distance. Pinhole acuity at distance was $6 / 18$ OS. Broad $\mathrm{H}$ testing showed no restrictions, no pain on eye movement, and no diplopia in any gaze. Amsler grid testing was normal OD; OS showed a scotoma in the inferior nasal quadrant. Red cap testing showed 75\% saturation OS compared to $100 \%$ saturation OD. Ishihara color testing was normal OD, but only the first plate was seen with OS. Pupils were equally round and reactive to light, with grade 1 RAPD in OS. Anterior segment evaluation by slit lamp was unremarkable. Angles by Van Herick measured 1:0.25. Intraocular pressures were $12 \mathrm{mmHg}$ OU with Goldmann Applanation Tonometry. Four-mirror gonioscopy was open to ciliary body in the inferior quadrant and scleral spur in the other quadrants OU. Dilated fundus examination showed grade 1 cortical cataract OU with trace posterior subcapsular cataract OS. Fundus examination (Figs. 1,2) using fundus biomicroscopy and binocular indirect ophthalmoscopy revealed clear vitreous and pink and distinct nerve with a cup to disc ratio of $0.65 \mathrm{OU}$. The nerves were large and the rim tissue was intact in both eyes. The optic nerve was negative for disc edema and pallor, and no signs of RNFL defects were observed OU. The optic nerve findings were consistent with what was measured on the SD-OCT (Fig. 3). The macula was flat and had even macular pigment; the vasculature showed a 2 to 3 ratio with normal course and caliber OU. The peripheral retina was negative for holes, tears, retinal detachment, or any pathology OU. The ganglion cell analysis (Fig. 4) and HD raster macula scans (Figs. 5,6) showed no loss of ganglion cells and no abnormalities of the macula OU. Humphrey Visual Field 30-2 (Figs. 7,8) was performed before dilation. The right eye was unremarkable with non-specific superior defects throughout the field. The left eye revealed a deep inferior altitudinal visual field defect and superior arcuate defect. The inferior defect was deeper than the superior defect. The visual field defect in the left eye did not match the clinical findings in the optic nerve, retina or OCT, suggesting a pathology posterior to the lamina.

An urgent referral for bloodwork including Erythrocyte Sedimentation Rate (ESR) and C-reactive Protein (CRP) was made to the family physician and a consult request was made to the on-call ophthalmologist to rule out a posterior ischemic optic neuropathy secondary to giant cell arteritis. MRI of the orbit and chiasm along with MRI of the neck was ordered to rule out any lesions more posterior or the possibility of carotid dissection. The minimal eye pain was to be re-evaluated once the cause of the vision loss was diagnosed.

Bloodwork revealed normal ESR, CRP and platelets. The on-call ophthalmologist saw the patient the next day. The exam findings were similar to those measured the day before, except that the patient's visual acuity in OS had further deteriorated to 20/100. Despite the lack of classic signs and symptoms, the patient was initially diagnosed with NA-AION by the ophthalmologist. No treatment was initiated at the time of investigation and vision slowly improved over the next 12 months. No optic nerve pallor or RNFL loss was noted at a one-year follow-up. The visual field defect was still present, but had improved. Further reflection on the case pointed to a diagnosis of PION, given the lack of clinical signs. The MRI results were unremarkable. 
Figure 1: Retinal photograph $O D$ at the time of the initial examination shows no disc edema, hemorrhage or pallor of the optic nerve, even pigment of the macula, and healthy vasculature.

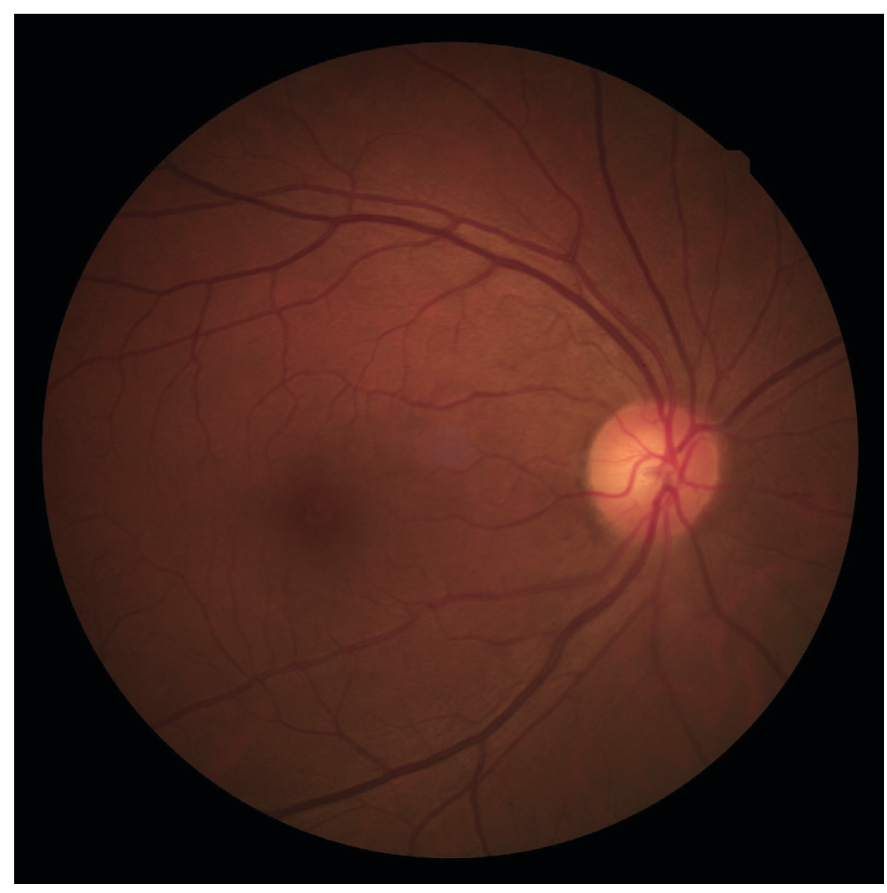

Figure 2: Retinal photograph OS at the time of the initial examination shows no disc edema, hemorrhage or pallor of the optic nerve, even pigment of the macula, and healthy vasculature.

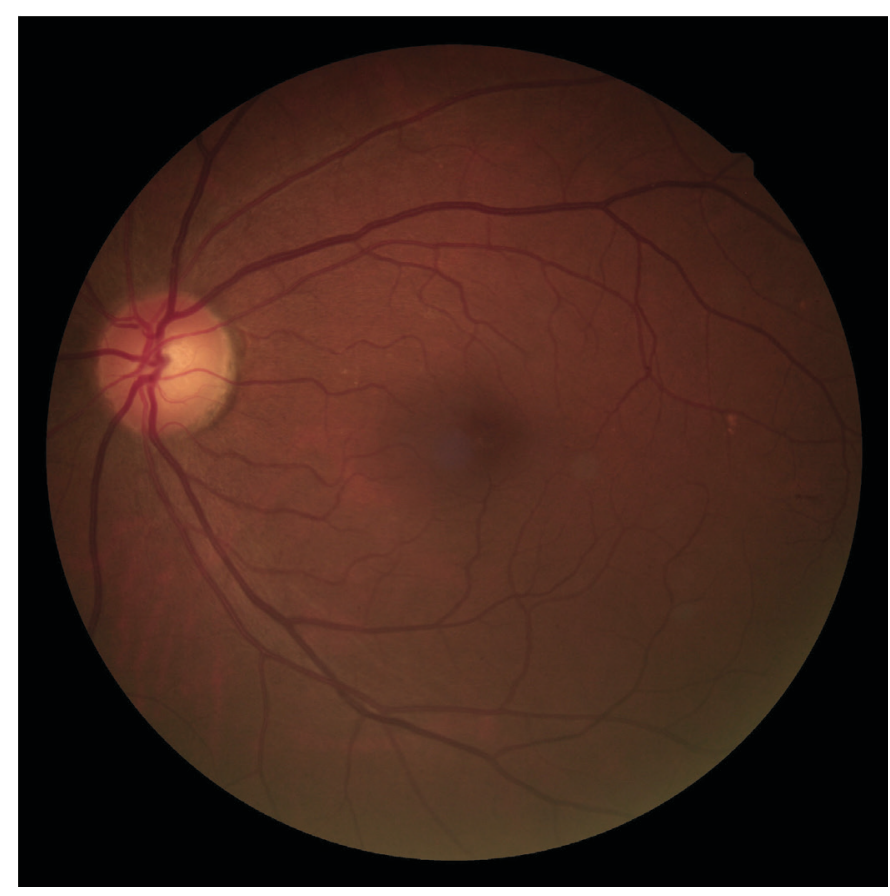


Figure 3: Zeiss Cirrus OCT Optic Nerve Head and RNFL OU at the initial examination shows healthy RNFL with no signs of optic nerve head edema $O U$.
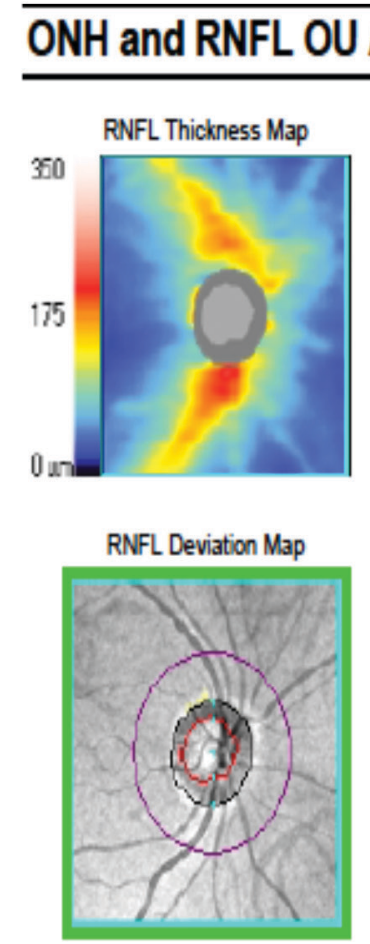

Disc Center(0.12,0.03)mm

Extracted Horizontal Tomogram

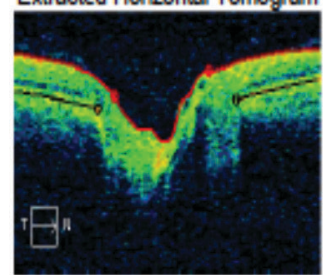

Extracted Vertical Tomogram

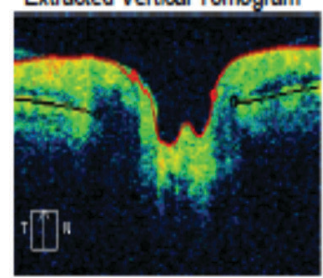

RNFL Circular Tomogram

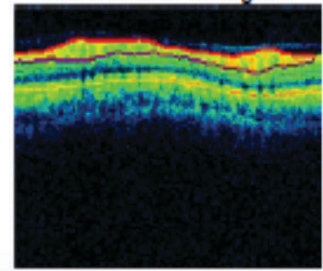

\begin{tabular}{|c|c|c|}
\hline$\Delta$ & OD & os \\
\hline Averase RNL Thisloness & $97 \mu \mathrm{m}$ & $103 \mu n$ \\
\hline Fifl Syminery & \multicolumn{2}{|c|}{$94 \%$} \\
\hline Rin Areo & $1.42 \mathrm{~mm}^{2}$ & $1.48 \mathrm{~mm}^{2}$ \\
\hline Cisc Ares & $247 m n^{2}$ & $2.63 \mathrm{~mm}^{2}$ \\
\hline Average C.D Rotio & Q.64 & 0.65 \\
\hline Yatical C.D Rotio & 060 & 0.60 \\
\hline Cup Volute & $0.250 \mathrm{~mm}^{3}$ & $0.255 \mathrm{~mm}^{3}$ \\
\hline
\end{tabular}

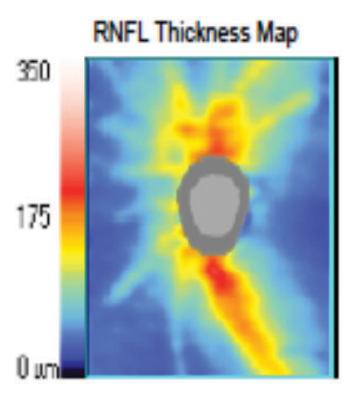

Neuro-retinal Rim Thickness

$\mu \mathrm{n} \quad-0 \mathrm{D} \ldots \mathrm{OS}$

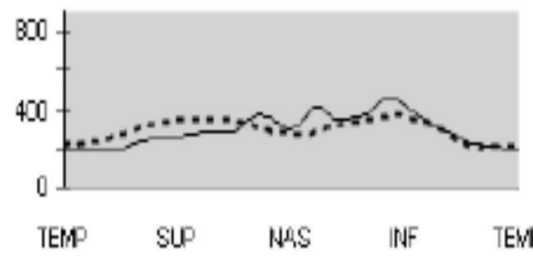

RNFL Thickness
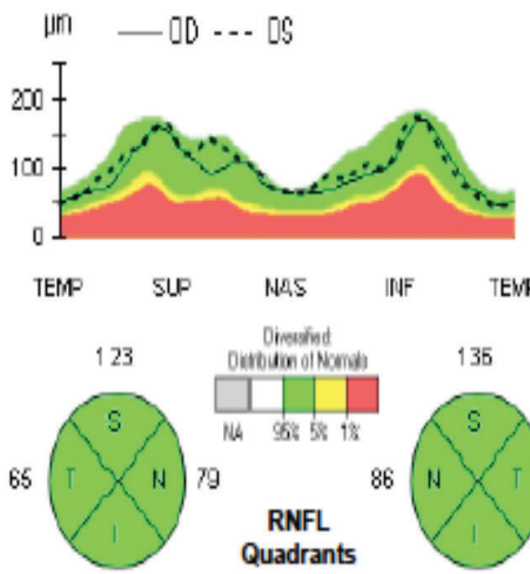

121

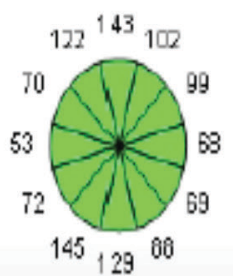

14512900

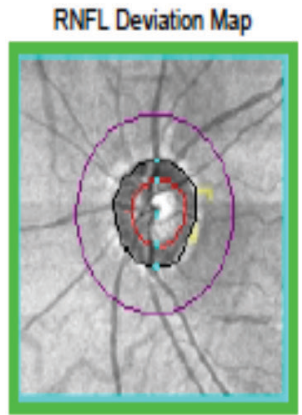

Disc Center $(0.03,0.27) \mathrm{mm}$ Extracted Horizontal Tomogram

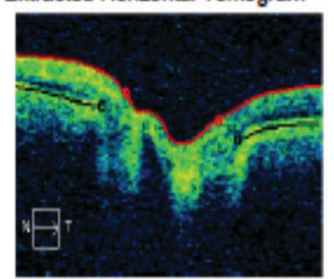

Extracted Vertical Tomogram

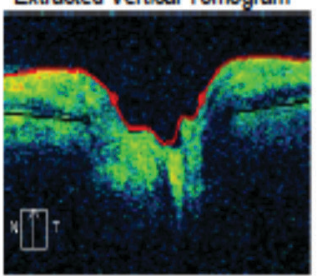

RNFL Circular Tomogram
RNFL

Clock

Hours

RNFL
Quadrants

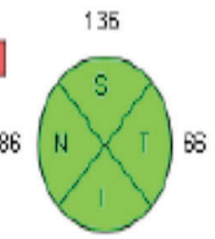

124

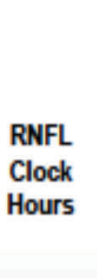

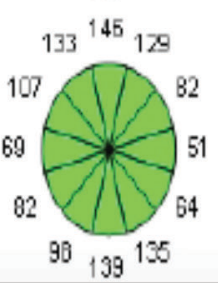

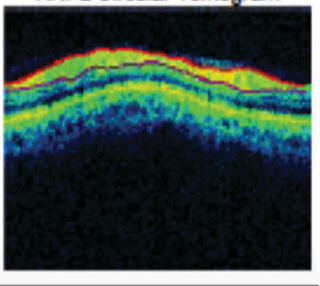


Figure 4: Zeiss Cirrus OCT Macula Cube (Ganglion Cell Layer Analysis) OU at the initial examination shows healthy GCC layer in both eyes. There is no hemispheric deterioration that may correlate to the visual field defect in the right eye.

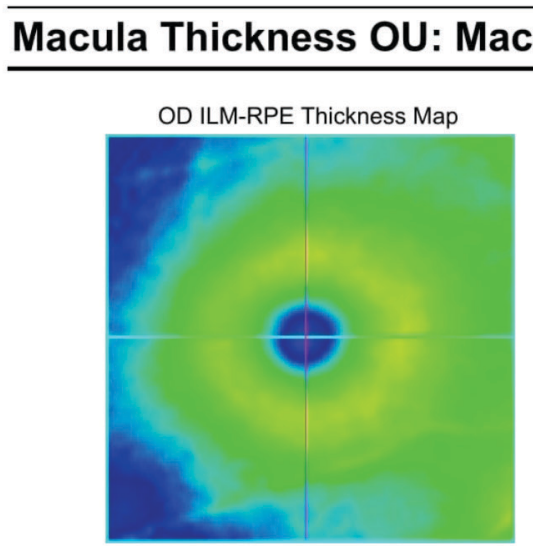

Fovea: 251,64

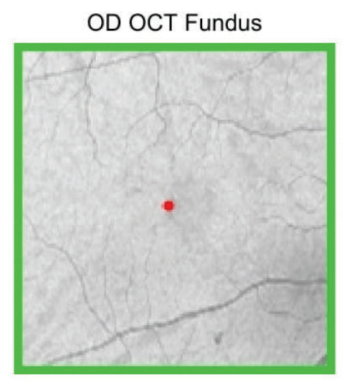

OD ILM-RPE Thickness
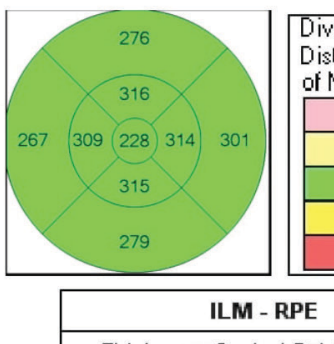

\begin{tabular}{|c|c|c|}
\hline ILM - RPE & OD & os \\
\hline Thickness Central Subtield ( $\mu m i)$ & 228 & 229 \\
\hline Volume Cube $\left(m+n^{3}\right)$ & 10.1 & 10.2 \\
\hline Thickness Avg Cube ( $\mu \mathrm{rn})$ & 281 & 284 \\
\hline
\end{tabular}

500
400
300
200
100
$0 \mu \mathrm{m}$
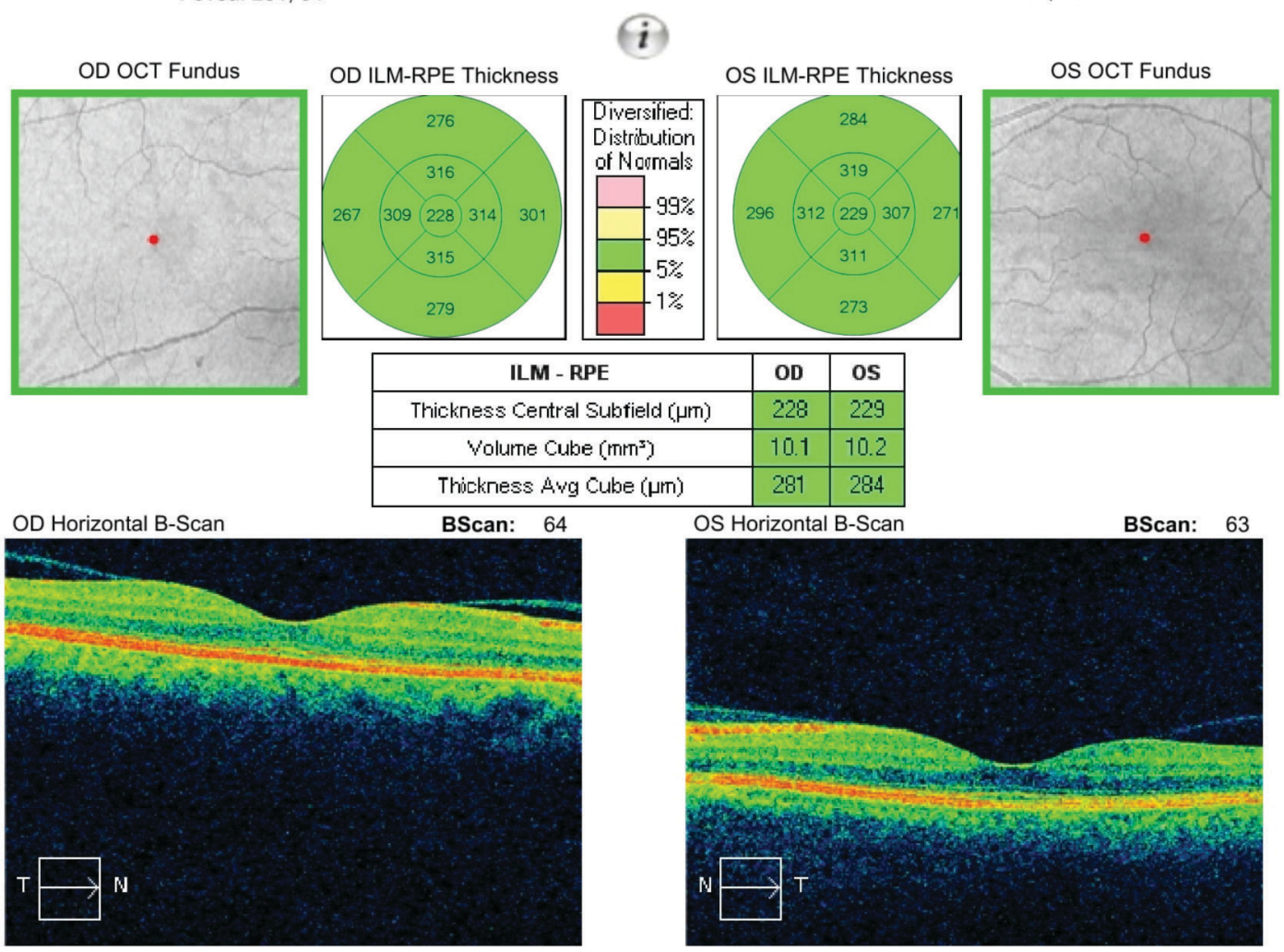
Figure 5: Zeiss Cirrus OCT HD raster (macula) OD at the initial examination shows healthy macula and no pathology that may be responsible for the deterioration of vision.

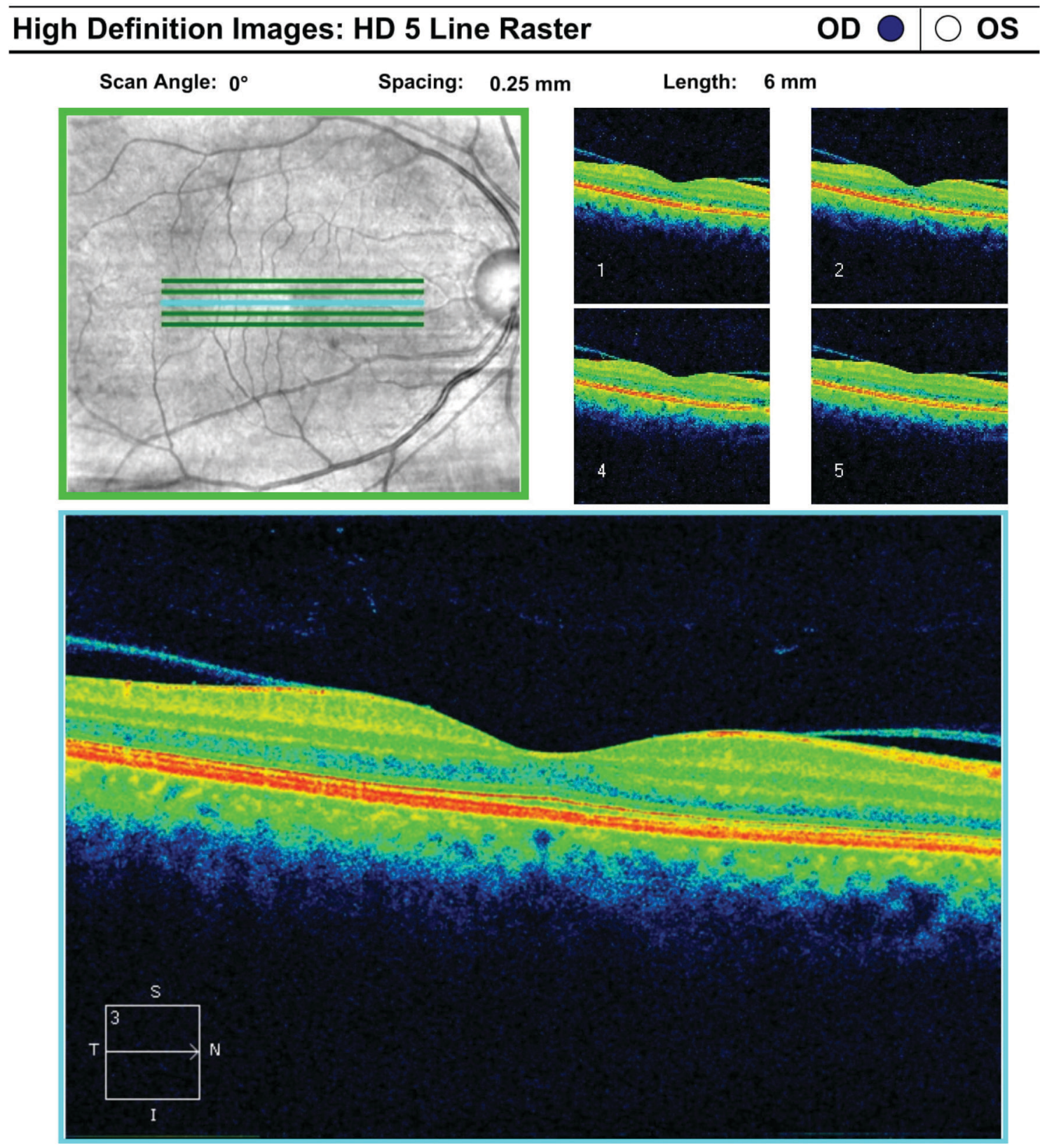


Figure 6: Zeiss Cirrus OCT HD raster (macula) OS at the initial examination shows healthy macula.

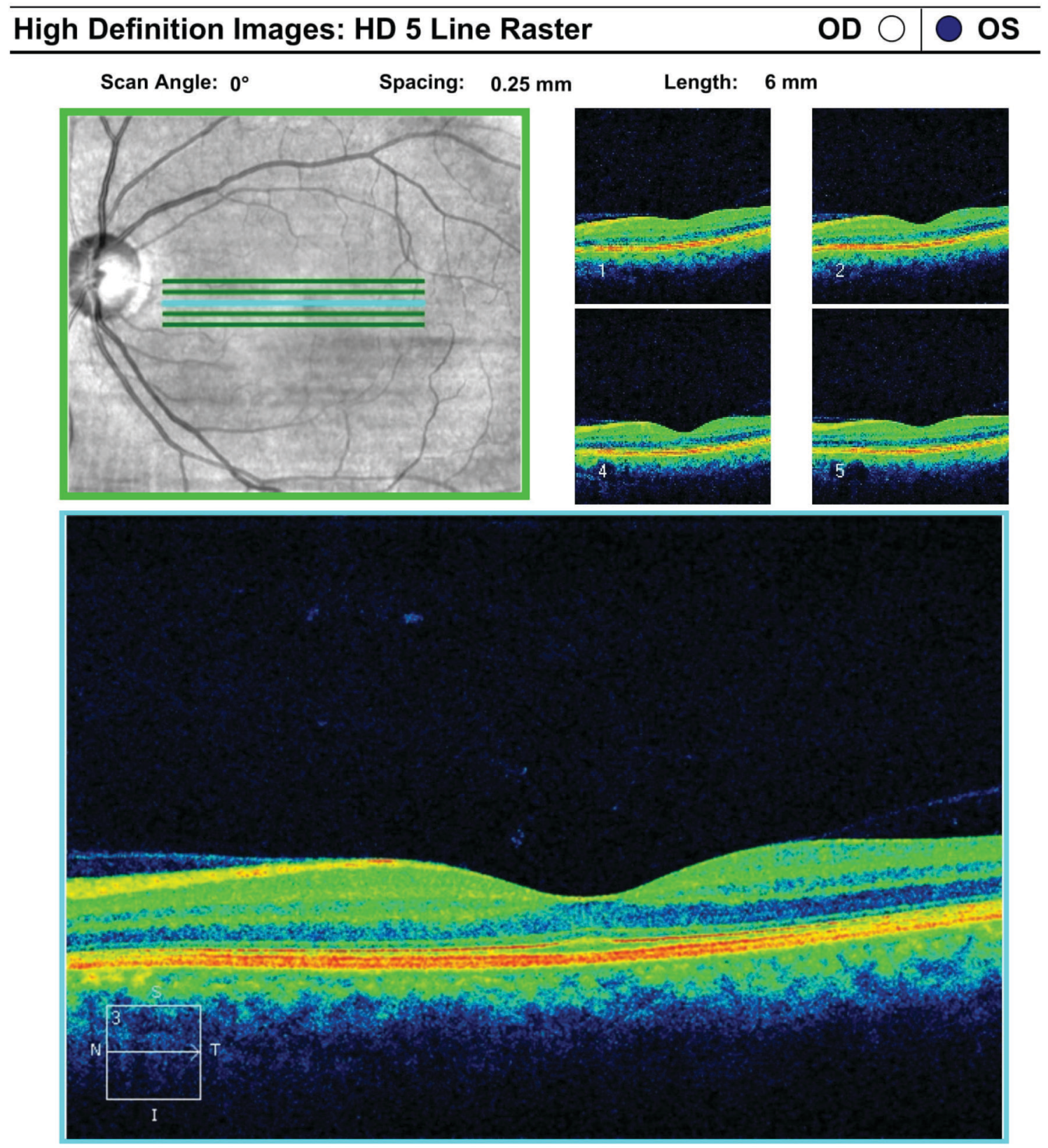


Figure 7: Humphrey Visual Field 30-2 SITA Standard OD at the follow-up shows mild scattered superior defects.

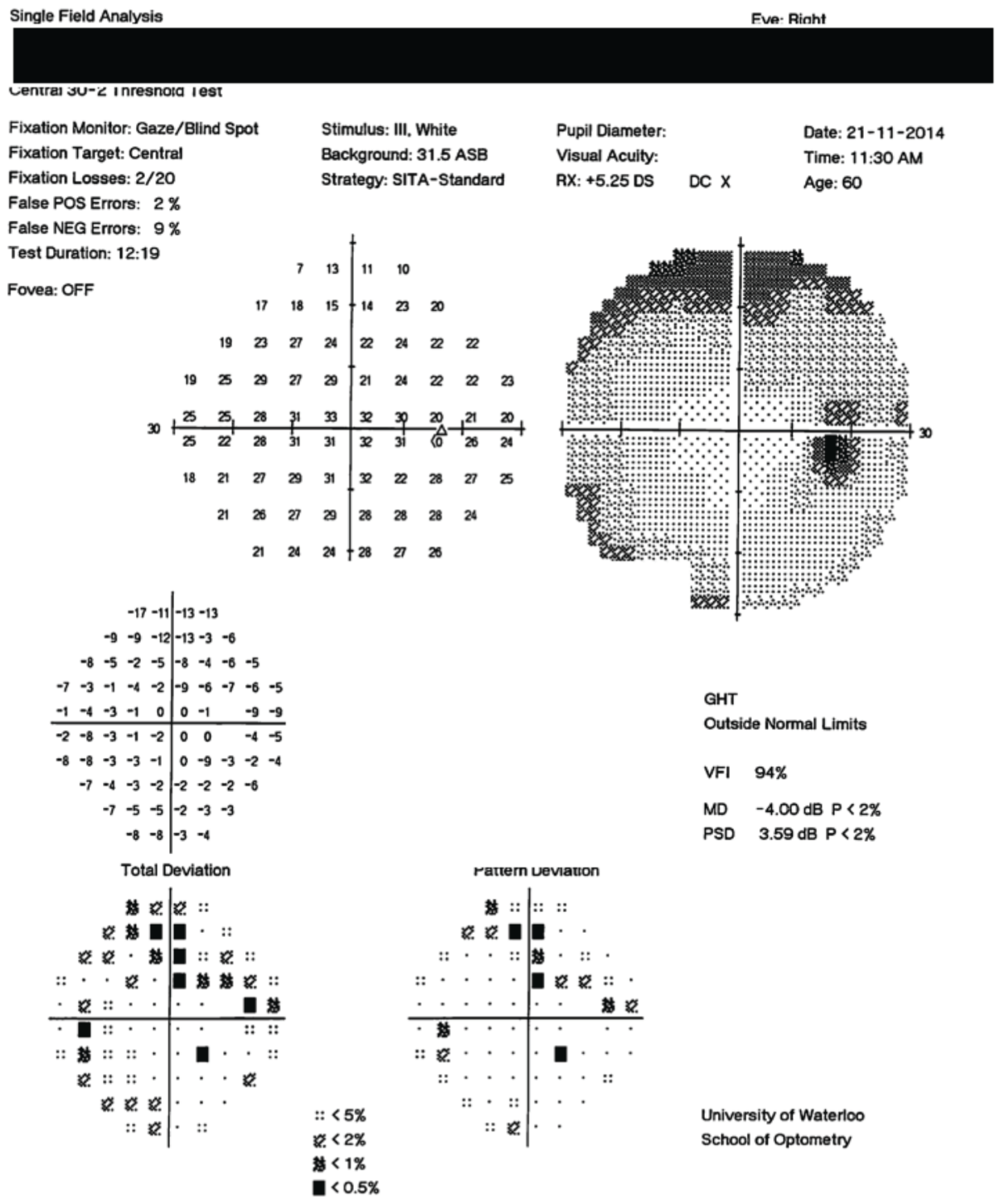

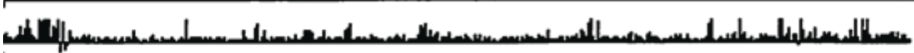

- 2010 Carl Zeiss Meditec

HFA II 740-40962-5.1.2/5.1.2 
Figure 8: Humphrey Visual Field 30-2 SITA Standard OS at the follow-up shows deep inferior altitudinal defect and superior arcuate defect.

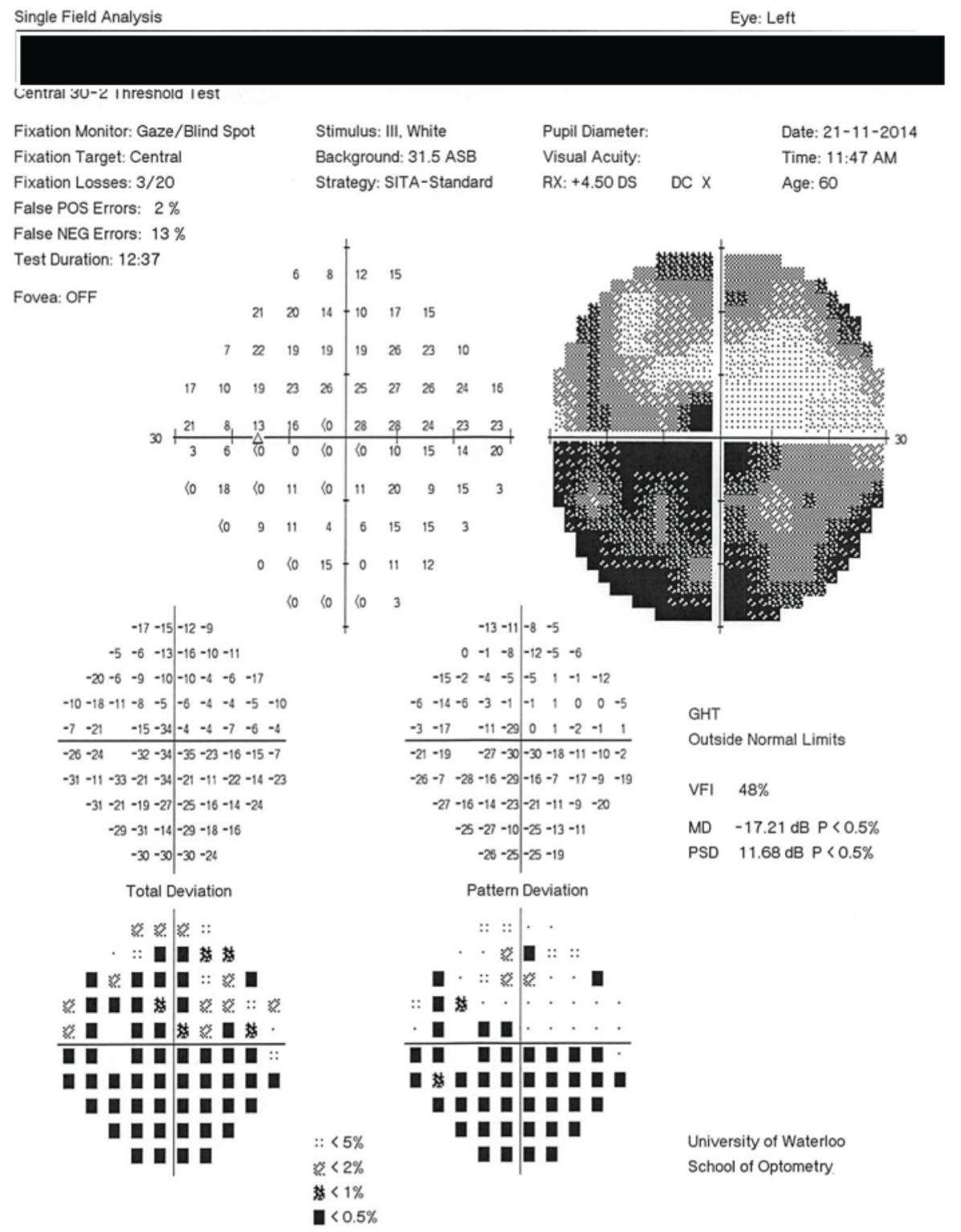

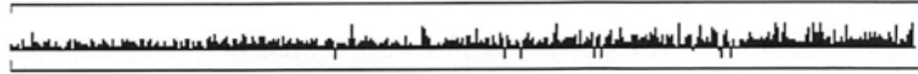

- 2010 Carl Zeiss Meditec

HFA II 740-40962-5.1.2/5.1.2 
DISCUSSION

Ischemic optic neuropathies can be differentiated based on the location of the ischemia. More commonly, the ischemia is in the anterior lamina and causes classical clinical signs in the optic nerve and vision. When the ischemia is behind the lamina, visual disruption occurs, but the optic nerve is left unaffected. AION and PION are similar in that they can be further classified into arteritic and non-arteritic etiologies.

To further understand PION, it is helpful to review the pathology of AION. AION is typically classified as arteritic (AAION) or non-arteritic (NA-AION). The difference between A-AION and NA-AION depends on whether the pathogenesis is secondary to vasculitis or other entities. ${ }^{5}$ A-AION is most commonly caused by giant cell arteritis (GCA), a specific type of vasculitis. Bloodwork including ESR and CRP, along with a thorough case history, is necessary to investigate the presence of inflammation if there is any suspicion of A-AION. Symptoms, including the presence of headache, scalp tenderness, as well as tenderness over the affected artery and jaw claudication, are often present in patients with GCA. In addition, myalgia, fatigue, weight loss, and decreased appetite are frequently seen. If GCA is suspected, immediate treatment is required. ${ }^{1,6}$ GCA is a true ocular emergency and permanent vision loss will ensue if it is not managed as soon as possible.

The pathogenesis of NA-AION remains unclear, but seems to encompass common sources of ischemic injury including, but not limited to, thromboembolism, systemic hypotension, and atherosclerotic occlusion. ${ }^{5}$ On the other hand, NA-AION is not due to inflammation, indicating that inflammatory markers would not be elevated in a lab test. In the case described here, ESR, CRP and platelets were normal, which ruled out A-AION. In addition, the visual field presentation was more consistent with NA-AION than with A-AION. The patient presented with unilateral inferior altitudinal visual field defect, which is a common presentation for NA-AION. ${ }^{7}$ Studies show that there is value in using OCT for the diagnosis of NA-AION. ${ }^{8}$ After acute axonal swelling and then thinning, both GCC and RNFL would show thickening and then thinning of the layers. ${ }^{8}$ Furthermore, the location and severity of the visual field defect significantly correlates with RNFL thinning. ${ }^{8}$ Therefore, both AAION and NA-AION should present with optic disc edema and either thickening (acute stage) or thinning (after resolution of the edema) of RNFL and GCC on OCT, which were absent in this case. ${ }^{8,9}$ Similarly, a retinal or other optic nerve pathology can also be ruled out as a cause of the visual field defect due to the normal macula and optic nerve OCT. ${ }^{10,11}$ The nerve fiber layer showed a 31-micron difference in the superior nasal, suggesting a mild amount of asymmetry between the right eye and left eye. The asymmetry in RNFL in the superior nasal clock and the lack of hemispheric thinning seen in ganglion cell analysis in the left eye does not explain the diffuse and deep scotoma in the visual field OS. ${ }^{8,12}$ Based on the patient's age, symptoms, clinical findings, and lab results, it may still be safe to assume that this was a non-arteritic ischemic event, however the location of the ischemia was likely posterior, suggesting a diagnosis of PION.

PION can be divided into three different types; arteritic, non-arteritic, and peri-operative. ${ }^{13}$ The exact epidemiology or prevalence of PION is unknown, but in a study by Hayreh, the median age of non-arteritic PION was 61.5 years and that of arteritic PION was 73.5 years. ${ }^{13}$ There is considerable overlap with AION in terms of symptoms, systemic association, diagnostic testing, and treatment protocol. As with AION, some of the risk factors include anemia, intraoperative hypotension, diabetes, obesity, and intraoperative blood loss, which can decrease the ocular perfusion pressure. ${ }^{14-16}$

To further understand why PION is a diagnosis by exclusion, it is helpful to review the vascular system of the optic nerve. The optic nerve head is supplied by the central retinal artery (CRA) and short posterior ciliary arteries (SPCA).$^{17}$ The posterior part of the nerve is supplied by the CRA in the centre and pial vascular plexus, which is responsible for the peripheral vascular system..$^{13}$ In AION, the ischemia occurs due to the SPCA. ${ }^{3}$ The SPCA is the end-point of the arterial system without any anastomoses to other arterial branches. ${ }^{13}$ No other blood vessels can compensate for the SPCA if they become ischemic. ${ }^{13}$ In the posterior part of the optic nerve, the pial vascular plexus originates from the peripapillary choroid, circle of Zinn and Haller, central retinal artery, and ophthalmic artery. ${ }^{13}$ The pial vascular plexus is not the end-point of the arterial system, so blood is able to freely relocate within the collateral plexus. This serves as a protective factor for the posterior part of the nerve if a region of a collateral system becomes ischemic. ${ }^{13}$ Therefore, there is less chance for ischemia to occur in the posterior part of the optic nerve.

Among NA-PION cases described by Hayreh, 24 of 35 eyes presented with a visual acuity of $20 / 100$ or worse. ${ }^{13}$ At the initial examination, our patient presented with a visual acuity of 20/70. However, the next-day assessment by an oph- 
thalmologist showed further deterioration of visual acuity to $20 / 100 .{ }^{13}$ In the study by Hayreh, all of the patients underwent an extensive ocular examination along with fluorescein angiography, magnetic resonance imaging, and neurological evaluation to exclude any other ocular, optic nerve, or neurological disorder. ${ }^{13}$ In this case, with mild ocular pain (3/10) and lack of optic disc edema, MRI was beneficial for ruling out retrobulbar optic neuritis secondary to multiple sclerosis. Further, demyelinating optic neuritis most commonly occurs in young women 20 to 45 years of age. ${ }^{18,19}$

Visual field defects in ischemic optic neuropathies can vary depending on which part of the RNFL is damaged. For PION, studies show that a central defect is the most common visual field defect, but other defects might be observed. ${ }^{1,13}$ The type of visual field defect is determined by which vascular system is affected..$^{20}$ In the retina, the papillomacular bundle of RNFL exits from the temporal aspect and makes up the central core of the optic nerve. ${ }^{1}$ The posterior part of the optic nerve is not supplied by the CRA, only by the terminal capillary ends from the pial vascular plexus. ${ }^{12}$ This terminal plexus is called the watershed zone. ${ }^{1}$ The watershed zone has been shown to be the most vulnerable region for ischemia. ${ }^{1,21}$ In instances of decreased perfusion, the watershed zone will be the first region to become ischemic because there is a lack of blood supply to the central core of the optic nerve. ${ }^{1}$ This results in the central visual field defect that is most commonly present in patients with PION. ${ }^{1}$ Since the pial vascular plexus supplies different parts of the posterior optic nerve, localized ischemia can show different types of visual field defect, depending on which area of the plexus is affected and where the affected optic nerve fibres originate in the retina. ${ }^{13}$ In fact, Hayreh reported that only 9 of 32 eyes in his study showed central defects and the rest manifested a variety of visual field defects. ${ }^{13}$ In our case, the patient did not have a central defect, but rather presented with an inferior altitudinal defect with some nasal scotoma. Based on the pathophysiology of PION, the inferior altitudinal defect seen in this patient is not impossible, but may be a rare form of a visual field defect.

The treatment for NA-PION is similar to that for NA-AION. According to Hayreh, systemic corticosteroid treatment usually results in significant improvements in visual acuity and visual field. ${ }^{22-24}$ In one study by Hayreh, a cohort of 696 consecutive eyes with NA-AION, seen within 2 weeks of onset, and initial visual acuity of 20/70 or worse was assigned to systemic corticosteroid treatment or non-treatment. ${ }^{22}$ Six months after the initial visit, $69.8 \%$ of patients in the corticosteroid treatment group showed an improvement in visual acuity, compared to only $37.1 \%$ in the nontreated group..$^{22}$ A similar comparison was done on improvements in visual field defects; after 6 months, $40.1 \%$ of the systemic corticosteroid-treated group showed improvement compared to only $24.5 \%$ of those who did not receive corticosteroid therapy. ${ }^{22}$ In these studies, corticosteroid treatment was continued until there was complete resolution of the optic disc edema. However, because PION does not present with optic disc edema, the benefits and duration of treatment with corticosteroids are still being investigated.

There is no definite follow-up protocol for PION. In one study, Hayreh initiated steroid treatment for 2 days, followed by rapid tapering and follow-up assessment in less than 2 weeks for both NA-PION and perioperative PION. ${ }^{13}$ However, long-term follow-up after initial onset varied from 1.3 to 214 months for NA-PION, 1.3 to 55.6 months for A-PION and 4.6 to 56.6 months for perioperative PION. Hayreh explained that the prognosis regarding visual recovery can also vary depending on the type of PION. In NA-PION, vision can be improved with high-dose corticosteroid therapy, whereas treatment of A-PION only prevents further vision loss. In NA-PION, spontaneous resolution of visual acuity and field may still occur without any treatment. In fact, only 5 of 16 treated eyes remained with stable vision, whereas 9 of 16 untreated eyes remained stable. ${ }^{13}$

\section{CONCLUSION}

Posterior ischemic optic neuropathy (PION)is a diagnosis of exclusion that can be made after a thorough patient history, presentation and appropriate diagnostic testing. This case shows the importance of co-management with ophthalmologists and family physicians in any case of suspected ischemic optic neuropathy, including suspected PION. As the primary eye care providers, optometrists need to be familiar with the differences between optic neuropathies, and how to find appropriate and prompt management for their patients. $\bullet$ 


\section{REFERENCES}

1. Sadda SR, Nee M, Miller NR, Biousse V, Newman NJ, Kouzis A. Clinical spectrum of posterior ischemic optic neuropathy. Am J Ophthalmol 2001;132(5):743-50.

2. Behbehani R. Clinical approach to optic neuropathies. Clin Ophthalmol 2007;1(3):233-46.

3. Atkins EJ, Bruce BB, Newman NJ, Biousse V. Treatment of nonarteritic anterior ischemic optic neuropathy. Surv Ophthalmol 2010;55(1):47-63.

4. Hayreh SS. Management of ischemic optic neuropathies. Indian J Ophthalmol 2011;59(2):123-36.

5. Patel HR, Margo CE. Pathology of ischemic optic neuropathy. Arch Pathol Lab Med 2017;141(1):162-6. doi: 10.5858/arpa.20160027-RS [doi].

6. Chacko JG, Chacko JA, Salter MW. Review of giant cell arteritis. Saudi J Ophthalmol 2015;29(1):48-52.

7. Han S, Jung JJ, Kim US. Differences between non-arteritic anterio ischemic optic neuropathy and open angle glaucoma with altitudinal visual field defect. Korean J Ophthalmol 2015;29(6):418-23. doi: 10.3341/kjo.2015.29.6.418 [doi].

8. Alasil T, Tan O, Lu AT, et al. Correlation of Fourier domain optical coherence tomography retinal nerve fiber layer maps with visual fields in nonarteritic ischemic optic neuropathy. Ophthalmic Surg Lasers Imaging 2008;39:S71-9.

9. Bellusci C, Savini G, Carbonelli M, Carelli V, Sadun AA, Barboni P. Retinal nerve fiber layer thickness in nonarteritic anterior ischemic optic neuropathy: OCT characterization of the acute and resolving phases. Graefes Arch Clin Exp Ophthalmol 2008;246(5):641-7.

10. Kim EK, Park HL, Park CK. Relationship between retinal inner nuclear layer thickness and severity of visual field loss in glaucoma. Sci Rep 2017;7(1):5543.

11. Suh W, Lee JM, Kee C. Depth and area of retinal nerve fiber layer damage and visual field correlation analysis. Korean J Ophthalmo 2014; 28(4):323-9.
12. Zhang X, Rasa AS, De Moraes GV, Hood DC. Mapping the visual field to retinal ganglion cell thickness image. Invest Ophthalmol Vis Sci 2011; 52:5085.

13. Hayreh SS. Posterior ischaemic optic neuropathy: Clinical features, pathogenesis, and management. Eye (Lond) 2004;18(11):1188-206.

14. Nickels TJ, Manlapaz MR, Farag E. Perioperative visual loss after spine surgery. World J Orthop 2014;5(2):100-6.

15. Epstein NE. Perioperative visual loss following prone spinal surgery: A review. Surg Neurol Int 2016;7(Suppl 13):347.

16. Cheng MA, Todorov A, Tempelhoff R, McHugh T, Crowder CM, Lauryssen C. The effect of prone positioning on intraocular pressure in anesthetized patients. Anesthesiology 2001;95(6):1351-5.

17. Cioffi GA. Ischemic model of optic nerve injury. Trans Am Ophthalmol Soc 2005;103:592-613

18. Dooley, MC, Foroozan R. Optic neuritis. J Ophthalmic Vis Res. 2010;5(3):182-7.

19. Dworak DP, Nichols J. A review of optic neuropathies. Dis Mon 2014;60(6):276-81.

20. Hayreh SS, Zimmerman MB. Optic disc edema in non-arteritic anterior ischemic optic neuropathy. Graefes Arch Clin Exp Ophthalmol 2007;245(8):1107-21

21. Hayreh SS. Posterior ciliary artery circulation in health and disease: the Weisenfeld lecture. Invest Ophthalmol Vis Sci 2004;45(3):74957. doi: 10.1167/iovs.03-0469 [doi].

22. Hayreh SS, Zimmerman MB. Non-arteritic anterior ischemic optic neuropathy: Role of systemic corticosteroid therapy. Graefes Arch Clin Exp Ophthalmol 2008;246(7):1029-46.

23. Hayreh SS, Zimmerman B, Kardon RH. Visual improvement with corticosteroid therapy in giant cell arteritis. report of a large study and review of literature. Acta Ophthalmol Scand 2002;80(4):355-67.

24. Hayreh SS, Zimmerman B. Visual deterioration in giant cell arteritis patients while on high doses of corticosteroid therapy. Ophthalmology 2003;110(6):1204-15. 